\title{
LOW COST ORIGAMI FABRICATION OF 3D SELF-ALIGNED HYBRID MICROFLUIDIC STRUCTURES
}

\author{
Janire Saez ${ }^{1}$, Lourdes Basabe-Desmonts ${ }^{2,3^{*}}$, Fernando Benito-Lopez ${ }^{1,4^{*}}$ \\ ${ }^{1}$ Microfluidics UPV/EHU Cluster, Analytical Chemistry Department, University of \\ the Basque Country UPV/EHU, Vitoria-Gazteiz, SPAIN \\ ${ }^{2}$ BIOMICs Research Group, Lascaray Ikergunea, Research Center, University of the \\ Basque Country, UPV/EHU, Vitoria, SPAIN \\ ${ }^{3}$ Ikerbasque, Basque Foundation for Science, 48011 Bilbao, SPAIN \\ ${ }^{4}$ Insight: Centre for Data Analytics, National Centre for Sensor Research, Dublin \\ City University, IRELAND
}

Corresponding authors: *lourdes.basabe@ehu.es *fernando.benito@ehu.es

\begin{abstract}
3D microfluidic device fabrication methods are normally quite expensive and tedious. In this paper we present an easy and cheap alternative wherein thin cyclic olefin polymer (COP) sheets, and pressure sensitive adhesive (PSA) were used to fabricate hybrid 3D microfluidic structures, by the Origami technique, which enables the fabrication of microfluidic devices without the need of any alignment tool. The COP and PSA layers were both cut simultaneously using a portable, low cost plotter allowing for rapid prototyping of a large variety of designs in a single production step. The devices were then manually assembled using the origami technique by simply combining COP and PSA layers and mild pressure. This fast fabrication method was applied, as proof of concept, to the generation of a micromixer with a 3D stepped serpentine design made of 10 layers in less than $8 \mathrm{~min}$. Moreover the micromixer was characterised as a function of its pressure failure, achieving pressures of up to 1000 mbar. This fabrication method is readily accessible across a large range of potential endusers, such as educational agencies (schools, universities), low income/developing world research and industry or any laboratory without access to clean room facilities, enabling the fabrication of robust, reproducible microfluidic devices.
\end{abstract}

Keywords Fabrication·Origami-COP-PSA Hybrid·Serpentine·Micromixer 


\section{Introduction}

Originally, developmental advances in microfluidic devices came from the microelectronics manufacturing sector. Traditionally, silicon was used as the base material for fabricating microfluidic devices for several applications (Lei 2014). The extensive study of silicon properties and its use in processing contributed to the rapid evolution of microfluidic technology. However, one of the main drawbacks of the use of silicon as fabrication material is that it is quite expensive, needs complicated microfluidic fabrication protocols and it is optically opaque to certain electromagnetic wavelengths, limiting its optical applications. To overcome these limitations, glass and polymer materials gained more significance in recent years to fabricate microfluidic devices. Compared to silicon, glass and polymer materials have the advantage of being cheap and optically transparent, although glass still require expensive and tedious fabrication protocols, such as photolithography and etching procedures, for the fabrication of microfluidic devices. As a result, polymers have become the preferred materials for microfluidic device production as they provide lower cost and simpler alternatives for fabrication, such as injection molding and hot embossing, which are more suitable for mass production. Some common used polymer materials include polymethylmethacrilate (PMMA), polydimethylsiloxane (PDMS), polystyrene (PS), polycarbonate (PC) or cyclic olefin polymer (COP). (Chen et al. 2014, Karlsson et al. 2012, Maitz 2015, Zhang et al. 2010)

Specifically, COP is becoming increasingly popular for the fabrication of microfluidic systems due to its advantageous properties. It shows magnificent properties such as being optically transparent and having high heat resistance with a glass transition temperature $(\mathrm{Tg})$ of $136{ }^{\circ} \mathrm{C}$ while still being a low cost material. COP has also the lowest water absorptivity of all plastics, endowing great dimensional stability under high humidity conditions upon the material. In addition to this, it is a chemically resistant and malleable material, and it can be chemically functionalised to perform surface assays (Gandhiraman et al. 2010). Moreover, this thermoplastic polymer has low degree of impurities, becoming a biocompatible material for biological, medical and environmental applications (RW.ERROR - Unable to find reference:138, BenitoLopez et al. 2014, Fuchiwaki and Nagai 2014, Harris et al. 2013, Moral-Vico et al. 2015, Tachibana et al. 2015). 
The most common microfluidic device fabrication methods for COP are injection molding, hot embossing, X-ray lithography and micromilling; but these methods are not necessarily accessible by the majority of researchers. Common limiting factors for these traditional methods are the cost of the equipment and that the fabrication requires highly specialised training and usually tedious, time consuming techniques such as the fabrication of molds and operation of the equipment itself in the case of injection molding for example. However, the expanding microfluidic community is seeking accessible, low-cost, and fast methods for rapid prototyping and fabrication of microfluidic devices. Fabrication methods for microfluidic devices using xurography (Bartholomeusz et al. 2005) followed by thermo-compression or chemical bonding, have gained gradual interest due to their relatively rapid way of fabrication. Xurography, which uses a cutting plotter (a plotter that holds a knife), traditionally used in the design industry for cutting graphics in adhesive vinyl films, is able to obtain very precise structures within the micrometer range, usually $\geq 200 \mu \mathrm{m}$.

Other rapid prototyping methods have been implemented for the fabrication of microfluidic devices as 3D printing (Au et al. 2015, Ho et al. 2015), computer numerical control micromachining (Sugioka et al. 2014, Ziaie et al. 2004), X-ray lithography (Maldonado and Peckerar 2016) and $\mathrm{CO}_{2}$ laser (Thompson et al. 2015). Micromachining (micrometer range) and X-ray lithography (nanometer range) generate features of very high resolution. For instance, in X-ray lithography the accuracy is of about $30 \mathrm{~nm}$, although new approaches are extending the resolution to $15 \mathrm{~nm}$ (Maldonado and Peckerar 2016). Moreover, micromachining usually generates a rough channel surface causing sometimes imperfect bonding. This is a common disadvantage shared with $\mathrm{CO}_{2}$ laser. These techniques are generally expensive since require special type of equipment and trained personnel. As a positive fact, it could be considered that many companies are starting to provide access to this type of technology, which will dramatically reduce the device fabrication cost in the near future. On the other hand, in the case of $3 \mathrm{D}$ printing, there is a technological boom coming at the moment with tens of publications appearing every month. The main drawback is that the technology is still in its infancy and, although equipment price is been reduced, the cost (printer + materials) is still elevated (higher resolution bigger prices, e.g. a $3 \mathrm{D}$ printer of $€ 7000$ generates channel resolutions of about $200 \mu \mathrm{m}$ ). Therefore it could be considered that a 
$3 \mathrm{D}$ printer having the same resolution than our xurography process is about 30 times more expensive.

In literature both monolithic or hybrid bonding methods can be found for the fabrication of COP microfluidic devices (Liu et al. 2012, Sanjay et al. , Sanjay et al. 2015). Among these methods, direct bonding methods such as solvent bonding and thermal fusion bonding are the most commonly used (Tsao et al. 2007). Extensively, solvent bonding was emerged as an important method for sealing cyclic olefin copolymer (COC) microchannels (Wallow et al. 2007).

PSA has appeared as an excelled material alternative for bonding or sealing microfluidic devices (Keimel 2003). PSA has a high degree of tack with the ability to quickly wet the surface to which it is applied and provides instant bonding at low to moderate pressures. On the other hand, PSAs possess sufficient cohesion and elasticity, so that despite their aggressive tackiness they can be handled with the fingers and removed from smooth surfaces without leaving any residue (Benedek 2004). The type of PSA chosen for microfluidic applications depends on its chemical composition and should be selected according to its proposed use (biocompatible, transparent, opaque, conductive, insulator, etc.). Medical grade PSAs are elected for bio-medical applications due to their biocompatibilities (Czech and Kowalczyk 2011). For instance, Yuen et al. described a low-cost rapid prototyping method using a desktop digital craft cutter. They applied rapid prototyping and xyz alignment for the fabrication of hybrid transparency and PSA films 3D microfluidic devices within minutes.(Yuen and Goral 2010) However, laser printer transparency films have poor wettability and a surface treatment with oxygen plasma was required.

It is indisputable the synergy between COP and PSA materials for the fabrication of microfluidic devices. A large number of papers can be found in literature explaining the fabrication of COP microfluidic devices where PSA was used for bonding or sealing de device in applications such as the development of a device for monitoring anticoagulants at the point of care (POC) (Harris et al. 2013) or in the fabrication of optofluidic valves (Garcia-Cordero et al. 2010). Nevertheless in all their fabrication protocols the different layers of COP and PSA were xurographied individually and stuck together manually or through a mold before bonding. 
In this way, the Origami technique, a concept previously described in paper-based microfluidic devices by Whitesides and co-workers (Martinez et al. 2007, Martinez et al. 2008), in chemistry (Kirby and Wheeler 2013) and in biotechnology "self-folding cell-laden microstructures" (Kuribayashi-Shigetomi et al. 2012) was further developed by (Elizalde et al. 2013) for COP and then applied by us for the fabrication of modular microvalve structures are of great interest (Benito-Lopez et al. 2014). The process consists of two steps: (1) folding of a layer of previously xurographied COP containing all the microfluidic structures and (2) thermocompression of the layers for the generation of the monolithic structure (Elizalde et al. 2013). For instance, in our previous publication, after the Origami fabrication of the 3D monolithic COP microfluidic device, a PSA layer was used to seal the device (microvalve). With this method a thermocompression step, using a high pressure and temperature device, is necessary for the fabrication of the 3D COP structure.

In this work we propose an easy, cheap, rapid and universal way for the fabrication of COP-PSA hybrid 3D microfluidic structures by the Origami technique without the use of a thermocompression step. The COP and PSA layers were cut using a portable, lowcost cutting plotter $(<€ 200)$. The layers were then assembled using the Origami technique combining the COP and PSA layers and the final 3D hybrid microfluidic structure was bonded using just mild pressure with a roller. This enables the fabrication of hybrid COP-PSA microfluidic devices at any type of laboratories by just acquiring a low price plotter and COP and PSA sheets. As a result, this technique is adaptive to any setting, for example income/ developing world regions or an educational setting making the fabrication protocol multidisciplinary. This fabrication technique was applied to the fabrication of an elaborate micromixer that consists of a 3D stepped serpentine design for proof of concept.

\section{Experimental}

\subsection{Experimental set-up and materials}

Zeonor COP sheets of $100 \mu \mathrm{m}$ thickness were purchased by Zeonex (Düsseldorf, Germany). ArCare ${ }^{\circledR} 8939$ PSA of $127 \mu \mathrm{m}$ double side roll was generously provided by Adhesive Research (Limerick, Ireland). 
A Silhouette Portrait ${ }^{\circledR}$ (Lehi, Utah, USA) was used as a cutting plotter for the cutting of the COP and PSA layers.

A Harvard Apparatus 11 elite syringe pump (Holliston, MA, USA) was used for the injection of the two liquids into the mixer. $1 \mathrm{~mL}$ syringes from Novica Medica (Barcelona, Spain) with precision tips from Nordson EFD (Bedfordshire, England) were connected to 1/16" ID Tygon tubing from ColmerPalmer (Vernon Hills, IL, USA). The tubes were connected to male luers that couple to female luers (ChipShop, Jena, Germany). Double side PSA circles (also cut with the same cutter plotter) were used to adhere and therefore to seal the female luers to the microfluidic devices and so prevent liquid leakage.

A rubber roller was purchased from Rotulatienda (Jerez, Cadiz, Spain).

The failure pressure of the microfluidic device was measured and controlled with a MSFC $^{\circledR}$ from Fluigent (Villejuif, France).

For the visual monitoring of the mixing, yellow and red food dyes (McCormick, Sabadell, Spain) were used.

\subsection{Channel dimensions and device fabrication}

The channel width is defined by the cutting process of the plotter. Therefore channels of different widths were cut: $1000,500,250,100,50$ and $25 \mu \mathrm{m}$. It was observed that the fabrication of channels smaller than $250 \mathrm{~mm}$ was not possible since all the generated channels were obtained with dimensions of $250 \pm 20 \mathrm{~mm}$. This is a limitation of the current fabrication technology.

During the assembly of the device, Origami technique, it was found out that the minimal channel dimensions that generated an operative microfluidic device was 500 $\mu \mathrm{m}$. Channels of $250 \mu \mathrm{m}$ did not get properly aligned during the origami process so the devices were inoperative.

\subsection{Micromixer performance}

In order to prove the fabrication method, a microfluidic device design was envisioned as a micromixer. The performance of the micromixer was carried out using yellow and red dye solutions injected into the microfluidic device using a syringe pump. The solutions 
were prepared by mixing $5 \mathrm{~mL}$ of water with $20 \mu \mathrm{L}$ of the corresponding food dye (yellow or red). The solutions were injected at a constant flow rate $\left(5 \mu \mathrm{L} \mathrm{min}{ }^{-1}\right)$ for 5 min and the visualisation of the mixing process of both dyes through the stepped serpentine was achieved by eye.

\section{Results and Discussion}

\subsection{Fabrication of the micromixer}

Generally, POC systems integrate microscale mixers to enhance biochemical interactions (Cosentino et al. 2015) or to speed up chemical reactions (Chen et al. 2016). Occasionally, it is convenient to have these micromixers as independent entities that can be coupled to the main microfluidic device following a modular approach. Therefore, as a proof of concept of our simple 3D fabrication technique, it was decided to develop a fabrication method of COP/PSA microfluidic structures to be used as passive micromixers.

After AutoCAD design of the 3D structures, both the COP and the PSA designs were cut in a single step with the Silhouette Portrait ${ }^{\circledR}$ plotter. The benefits of using this type of equipment are its portability (less than $1.6 \mathrm{~kg}$ and $20 \mathrm{x} 40 \mathrm{~cm}$ dimensions) and its extremely low-cost $(<€ 200)$. Moreover the plotter allows laboratories without access to clean room facilities or with low resources the ability to rapidly design, fabrication and subsequent tests of microfluidic devices for a huge variety of applications. The plotter is able to generate structures, microfluidic channels, down to $500 \mu \mathrm{m}$ in width in both COP and PSA of different thicknesses.

Micromixers are generally designed with channel geometries that decrease the mixing path and increase the contact surface area (Capretto et al. 2011). The micromixer design was visualised as a 3D stepped serpentine. The 3D design was sliced into five 2D layers of COP and another five 2D layers of PSA, separating the different sections by a dotted line which ensures the precise bending of the layers. The 3D serpentine was envisioned with four steps connected by channels. Each of the channels has a width of $1 \mathrm{~mm}$ and a length of $8 \mathrm{~mm}$ with the two inlets forming a T-shape, as illustrated in Figure 1. 


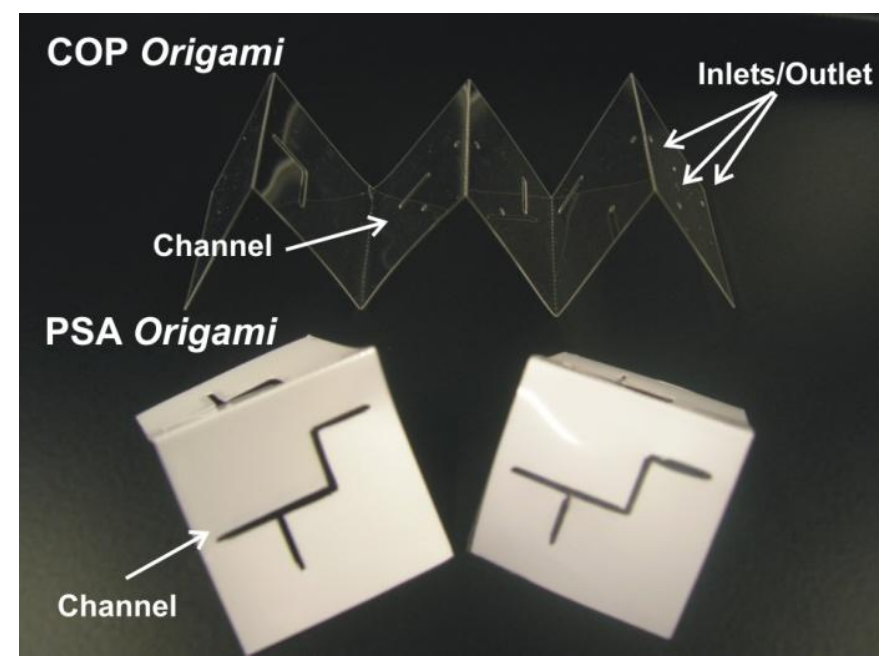

Figure 1: COP and PSA layers after xurography and bending (following the dotted lines) showing the inlets/outlet and channels in detail.

COP or PSA sheets were placed on the plotter carrier-sheet and a slight pressure was applied with the roller to ensure that the sheets remained fixed to the carrier sheet during the entire cutting process, Figure 2. The COP or PSA sheets were cut and the excised material was removed by scratching with a precision tip.

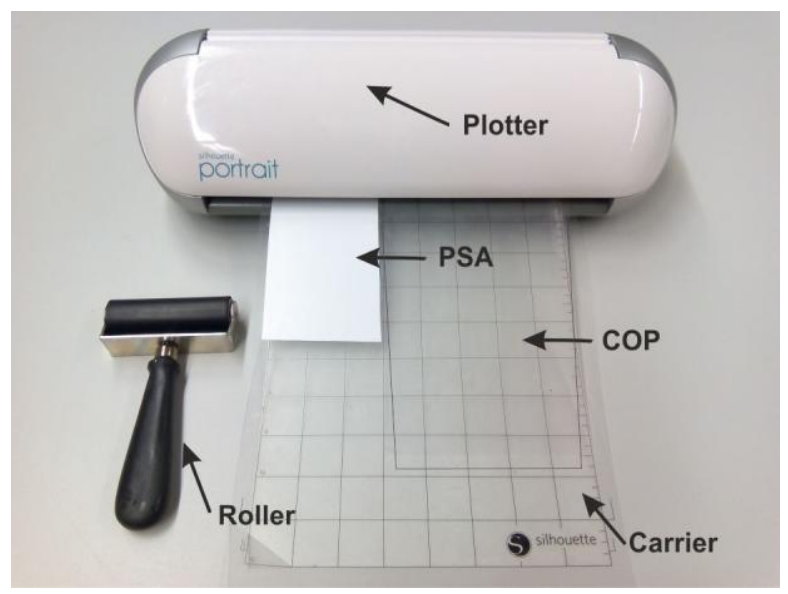

Figure 2: Silhouette Portrait plotter set-up for rapid prototyping and roller.

Once the COP and PSA sheets were cleaned with air and folded along the dots lines as shown in Figure 2, the folded double side PSA layers were used as bonding agents for the COP sections, as represented in Figure 3, following this protocol:

1) One of the PSA layers is able to bond three sections of the COP layer. Careful folding must be carried out to ensure that no air bubbles or wrinkles in the COP or in the double sided PSA get trapped during the process. 
2) The other PSA layer bonds to the other three sections of COP layer, on the opposite side of the COP layer.

3) Then the last available section of the PSA layer (from step two) bonds together the two sides of the COP layer.

4) The device was totally assembled. The device alignment was achieved by using two crystals to sandwich the layers and a microscope.

The manual assembly of the different sections of the channels caused minor misalignment in the final channel of the device. The width of the channel was calculated to be in the $\mathrm{Y}$ axis $944.11 \pm 1.47 \mu \mathrm{m}(\mathrm{n}=3)$ and in the $\mathrm{X}$ axis $938.17 \pm 1.46 \mu \mathrm{m}(\mathrm{n}=3)$, which is in the same range as misalignments generated during the fabrication of the monolithic 3D COP devices proposed by Elizalde et al (Elizalde et al. 2013).

The final micromixer is composed of six layers of COP, $100 \mu \mathrm{m}$ thickness each, and five layers of PSA $127 \mu \mathrm{m}$ thickness each, with an area of $2.5 \mathrm{~cm}^{2}$ and $1.135 \mathrm{~mm}$ in total thickness as illustrated in Figure 4.
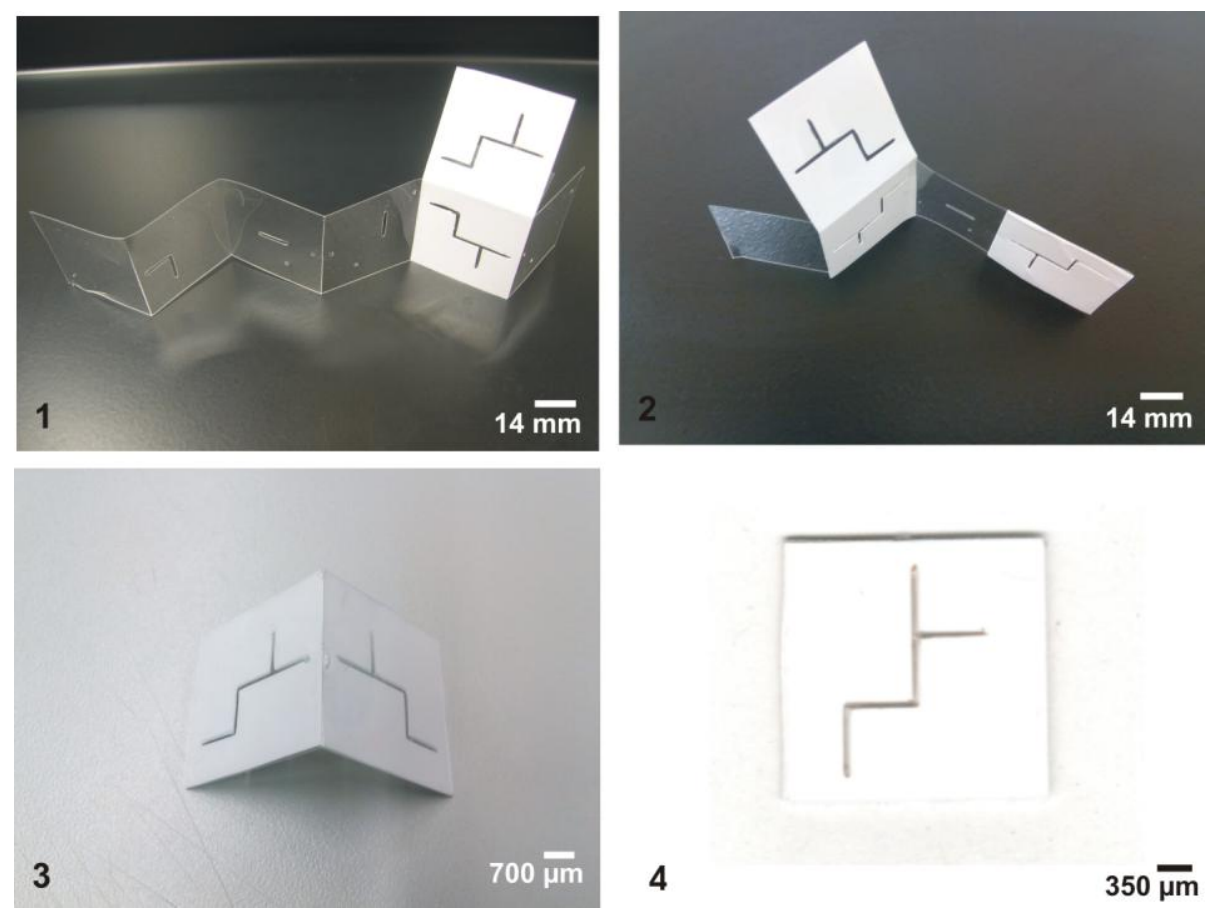
Figure 3: Set of pictures showing 1) PSA bonds to one COP section. 2) PSA folds and bonds to the other side of the COP section 3) Both sides bonded by PSA 4) micromixer assembled.

Although the micromixer was fabricated as a modular entity that can be used independently, the same fabrication protocol could be applied for the fabrication of fully functional microfluidic devices. Moreover since the fabrication protocol uses biocompatible materials, makes it very interesting for fast prototyping of microfluidic devices for POC applications in laboratories that do not have access to microfabrication facilities.

The advantages of the different bonding techniques existing in literature for COP xurography, compared with this new origami technique, are summarised in Table 1.

Table 1: Advantages of the different bonding techniques for COP xurography.

Bonding Method

\begin{tabular}{|c|c|c|c|}
\hline Advantage & $\begin{array}{c}\text { Solvent } \\
\text { (Elizalde et al. 2013, Mair et al. } \\
\text { 2007, Nunes et al. 2010, Ogilvie et } \\
\text { al. 2010) } *\end{array}$ & $\begin{array}{l}\text { Thermocompression } \\
\text { (Elizalde et al. 2013, Nunes et } \\
\text { al. 2010, Tsao et al. 2007) * }\end{array}$ & COP/PSA origami \\
\hline Low cost & $\mathrm{X}$ & $\mathrm{X}$ & $\sqrt{ }$ \\
\hline Low power & $\sqrt{ }$ & $X$ & $\sqrt{ }$ \\
\hline $\begin{array}{c}\text { Portable } \\
\text { Fast }\end{array}$ & $X$ & $X$ & $\sqrt{ }$ \\
\hline $\begin{array}{l}\text { fabrication } \\
\text { times }\end{array}$ & $\mathrm{X}$ & $\mathrm{X}$ & $\sqrt{ }$ \\
\hline $\begin{array}{c}\text { Clean room } \\
\text { free }\end{array}$ & $X$ & $\mathrm{X}$ & $\sqrt{ }$ \\
\hline Bonding steps & 4 & 3 & 2 \\
\hline $\begin{array}{c}\text { Equipment } \\
\text { needed }\end{array}$ & Heater/Pressure & Heater & None \\
\hline
\end{tabular}

\subsection{Characterisation of the micromixer}

To determine the mixing performance two colored liquids, yellow and red, were mixed. A standard double syringe pump with two $1 \mathrm{~mL}$ syringes were used to introduce the liquids at $1,3,5,7,15$ and $20 \mu \mathrm{L} \mathrm{min}^{-1}$ respectively in the micromixer. The results 
showed that for any of the flows applied a very uniform mixing, along the channel, was achieved by the observation of a homogeneous orange colour at the outlet. The mixing was achieved even at high flows $\left(20 \mu \mathrm{L} \mathrm{min}^{-1}\right)$, see Figure 4 (left):

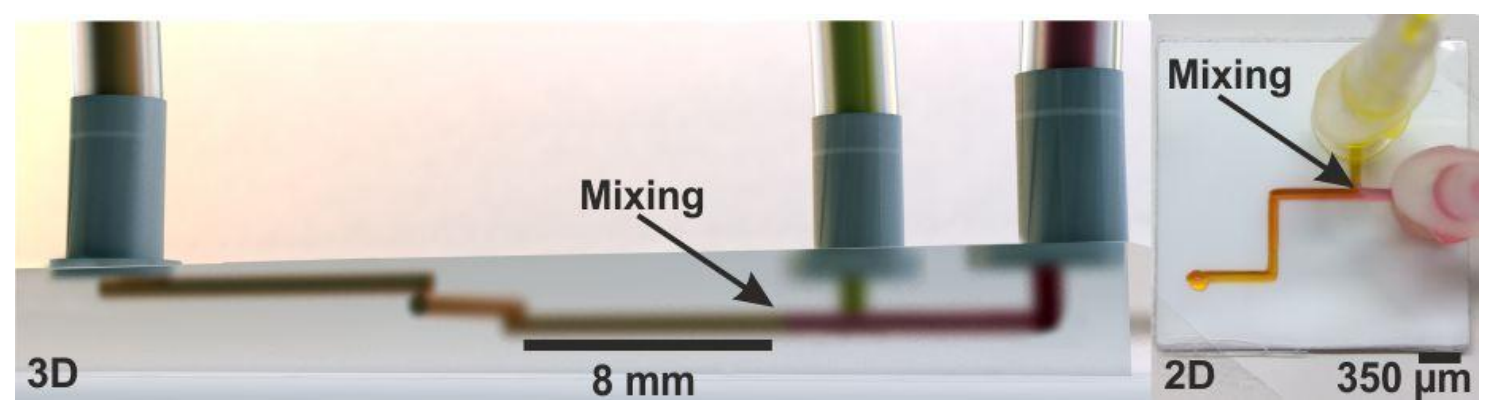

Figure 4: Scheme of the micromixer in 3D view (left) and a $2 D$ view of the micromixer performance when introducing the red and yellow dye solutions at $20 \mu \mathrm{L} \mathrm{min}^{-1}$ (right).

At the beginning of the injection, first step of the micromixer, the laminar flow of the two solutions was appreciable at high flow rates. Nevertheless, this phenomenon rapidly disappears at the beginning of the second step (after the $8 \mathrm{~mm}$ channel), when both solutions get in contact and mix when jumping to the second step of the micromixer Figure 4 (right). During the third and the fourth step the colour of the solution is completely mixed and homogeneous (orange).

In order to evaluate the strength of the bonding process during Origami bending, pressure robustness tests were carried out with the micromixers. In order to do that, two reservoirs of $1 \mathrm{~mL}$ volume were filled with deonised water and 50, 100, 500 and 1000 mbar of pressure were applied with the MSFC $^{\circledR}$, see Figure 5. This protocol was applied several times to each of the devices to ensure their reusability at those pressures. It was found out that all the micromixers could hold up to 1000 mbar $(n=5 ; 5$ different devices) of pressure with a hydrodynamic resistance of $1.15 \mathrm{~Pa} \mathrm{~s} \mathrm{~m}^{-2}$ without any damage. None of the devices tested suffered any damage during the pressure tests. Moreover, all the devices were reused many times ( $>8$ times) at atmospheric pressure without losing their functionality. 


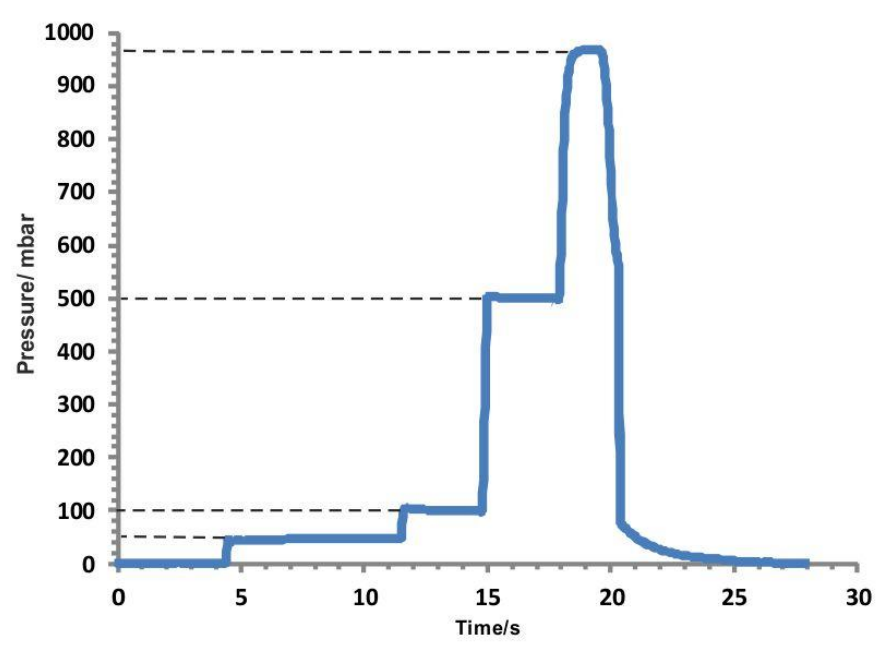

Figure 5: a) Pressures that the micromixer can hold: steps at 50, 100, 500 and 1000 mbar, respectively. The drop of the signal at time $20 \mathrm{~s}$ is due to the emptying of the $1 \mathrm{~mL}$ reservoir.

\section{Conclusions}

An easy and fast fabrication method of hybrid 3D COP-PSA microfluidic structures by the Origami technique is shown in this work. The devices were cut by a Silhouette Portrait plotter, a very cheap equipment alternative, which is portable and allows the fast fabrication of microfluidic structures for different applications at any setting. The assembly of the devices was carried out using the Origami technique combining layers of COP and PSA that bent and bonded to each other as an Origami. No thermocompression was required and just a soft compression with a hand roller was necessary. This fabrication protocol was used for the generation of a 3D stepped serpentine micromixer. The micromixers were found to be efficient even at high flows $\left(20 \mu \mathrm{L} \mathrm{min}{ }^{-1}\right)$ and able to stand pressures up to 1000 mbar.

\section{Acknowledgements}

Fernando Benito-Lopez acknowledges the Ramón y Cajal Programme (Ministerio de Economía y Competitividad), Spain. This project has received funding from the European Union's Seventh Framework Programme (FP7) for Research, Technological Development and Demonstration under grant agreement no. 604241. JS and FBL personally acknowledge Marian Martínez de Pancorbo for let them use her laboratory facilities at UPV/EHU. Authors also acknowledge Adhesive Research for the donation of the PSA samples and to Iñaki Veci for the drawing of the 3D scheme. 


\section{References}

Au AK, Bhattacharjee N, Horowitz LF, Chang TC, Folch A (2015) 3D-printed microfluidic automation. Lab Chip 15:1934-1941

Bartholomeusz D,A., Boutté R,W., Andrade J,D. (2005) Xurography: rapid prototyping of microstructures using a cutting plotter. IEEE/ASME Journal of Microelectromechanical Systems 14:1364-1374

Benedek I (2004) Pressure sensitive adhesives and applications. Marcel Dekker, Inc., New York

Benito-Lopez F, Antoñana-Díez M, Curto VF, Diamond D, Castro-López V (2014) Modular microfluidic valve structures based on reversible thermoresponsive ionogel actuators. Lab Chip Miniaturisation Chem Biol 14:3530-3538

Capretto L, Cheng W, Hill M, Zhang X (2011) Micromixing within microfluidic devices. Top Curr Chem 304:27-68

Chen P, Chen Y, Tsai C (2016) Microfluidic chip for rapid mixing and uniform distribution of multiple reagents using commercial pipettes. Microelectronic Engineering 150:57-63

Chen Y-, Cheng Y-, Kim HS, Ingram PN, Nor JE, Yoon E (2014) Paired single cell coculture microenvironments isolated by two-phase flow with continuous nutrient renewal. Lab Chip Miniaturisation Chem Biol 14:2941-2947

Cosentino A, Madadi H, Vergara P, Vecchione R, Causa F, Netti PA (2015) An efficient planar accordion-shaped micromixer: from biochemical mixing to biological application. Sci Rep 5:1-10

Czech Z, Kowalczyk A (2011) Pressure-Sensitive Adhesives for Medical Applications. In: . Dr. Isin Akyar. http://www.intechopen.com/books/howtoreference/wide-spectra-ofquality-control/pressure-sensitive-adhesives-for-medical-applications. Accessed 06/22 2016

Elizalde J, Antoñana M, Matthys L, Laouenan F, Ruano-López JM (2013) Rapid prototyping of self aligned 3D microfluidic structures. Int Conf Miniaturized Syst Chem Life Sci , MicroTAS 2:790-792

Fuchiwaki Y, Nagai H (2014) Study of a liquid plug-flow thermal cycling technique using a temperature gradient-based actuator. Sensors (Basel) 14:20235-20244

Gandhiraman RP, Volcke C, Gubala V, Doyle C, Basabe-Desmonts L, Dotzler C, Toney MF, Iacono M, Nooney RI, Daniels S, James B, Williams DE (2010) High efficiency amine functionalization of cycloolefin polymer surfaces for biodiagnostics. $\mathrm{J}$ Mater Chem 20:4116-4127 
Garcia-Cordero JL, Kurzbuch D, Benito-Lopez F, Diamond D, Lee LP, Ricco AJ (2010) Optically addressable single-use microfluidic valves by laser printer lithography. Lab Chip 10:2680-2687

Harris LF, Rainey P, Castro-Lopez V, O'Donnell JS, Killard AJ (2013) A microfluidic anti-Factor Xa assay device for point of care monitoring of anticoagulation therapy. Analyst 138:4769-4776

Ho CMB, Ng SH, Li KHH, Yoon Y (2015) 3D printed microfluidics for biological applications. Lab Chip 15:3627-3637

Karlsson JM, Haraldsson T, Carlborg CF, Hansson J, Russom A, Van Der Wijngaart W (2012) Fabrication and transfer of fragile 3D PDMS microstructures. J Micromech Microengineering 22

Keimel FA (2003) Historical Development of Adhesives and Adhesives Bonding. In: Pizzi A, Mittal KL (eds) Handbook of Adhesive Technology, pp 1-12

Kirby AE, Wheeler AR (2013) Microfluidic origami: A new device format for in-line reaction monitoring by nanoelectrospray ionization mass spectrometry. Lab Chip Miniaturisation Chem Biol 13:2533-2540

Lei KF (2014) Materials and Fabrication Techniques for Nano- and Microfluidic Devices. In: Microfluidics in Detection Science: Lab-on-a-chip Technologies. RSC Detection Science, pp 1-28

Liu K, Gu P, Hamaker K, Fan ZH (2012) Characterization of bonding between poly(dimethylsiloxane) and cyclic olefin copolymer using corona discharge induced grafting polymerization. J Colloid Interface Sci 365:289-295

Mair DA, Rolandi M, Snauko M, Noroski R, Svec F, Fréchet JMJ (2007) RoomTemperature Bonding for Plastic High-Pressure Microfluidic Chips. Anal Chem 79:5097-5102

Maitz MF (2015) Applications of synthetic polymers in clinical medicine. Biosurface and Biotribology 1:161-176

Maldonado JR, Peckerar M (2016) X-ray lithography: Some history, current status and future prospects. Microelectron Eng 161:87-93

Martinez AW, Phillips ST, Whitesides GM (2008) Three-dimensional microfluidic devices fabricated in layered paper and tape. Proc Natl Acad Sci U S A 105:1960619611

Martinez AW, Phillips ST, Butte MJ, Whitesides GM (2007) Patterned paper as a platform for inexpensive, low-volume, portable bioassays. Angew Chem Int Ed 46:1318-1320

Moral-Vico J, Barallat J, Abad L, Olivé-Monllau R, Muñoz-Pascual FX, Galán Ortega A, del Campo FJ, Baldrich E (2015) Dual chronoamperometric detection of enzymatic 
biomarkers using magnetic beads and a low-cost flow cell. Biosensors and Bioelectronics 69:328-336

Nunes PS, Ohlsson PD, Ordeig O, Kutter JP (2010) Cyclic olefin polymers: emerging materials for lab-on-a-chip applications. Microfluid Nanofluidics 9:145-161

Ogilvie IRG, Sieben VJ, Floquet CFA, Zmijan R, Mowlem MC, Morgan H (2010) Reduction of surface roughness for optical quality microfluidic devices in PMMA and COC. J Micromech Microengineering 20

Sanjay ST, Fu G, Dou M, Xu F, Liu R, Qi H, Li X Biomarker detection for disease diagnosis using cost-effective microfluidic platforms. Analyst 140:7062-7081

Sanjay ST, Fu G, Dou M, Xu F, Liu R, Qi H, Li X (2015) Biomarker detection for disease diagnosis using cost-effective microfluidic platforms. Analyst 140:7062-7081

Sugioka K, Xu J, Wu D, Hanada Y, Wang Z, Cheng Y, Midorikawa K (2014) Femtosecond laser 3D micromachining: a powerful tool for the fabrication of microfluidic, optofluidic, and electrofluidic devices based on glass. Lab Chip 14:34473458

Tachibana H, Saito M, Shibuya S, Tsuji K, Miyagawa N, Yamanaka K, Tamiya E (2015) On-chip quantitative detection of pathogen genes by autonomous microfluidic PCR platform. Biosens Bioelectron 74:725-730

Thompson BL, Ouyang Y, Duarte GR, Carrilho E, Krauss ST, Landers JP (2015) Inexpensive, rapid prototyping of microfluidic devices using overhead transparencies and a laser print, cut and laminate fabrication method. Nat Protoc 10:875-886

Tsao CW, Hromada L, Liu J, Kumar P, DeVoe DL (2007) Low temperature bonding of PMMA and COC microfluidic substrates using UV/ozone surface treatment. Lab Chip 7:499-505

Wallow TI, Morales AM, Simmons BA, Hunter MC, Krafcik KL, Domeier LA, Sickafoose SM, Patel KD, Gardea A (2007) Low-distortion, high-strength bonding of thermoplastic microfluidic devices employing case-II diffusion-mediated permeant activation. Lab Chip 7:1825-1831

Yuen PK, Goral VN (2010) Low-cost rapid prototyping of flexible microfluidic devices using a desktop digital craft cutter. Lab Chip Miniaturisation Chem Biol 10:384-387

Zhang M, Wu J, Wang L, Xiao K, Wen W (2010) A simple method for fabricating multi-layer PDMS structures for 3D microfluidic chips. Lab Chip Miniaturisation Chem Biol 10:1199-1203

Ziaie B, Baldi A, Lei M, Gu Y, Siegel RA (2004) Hard and soft micromachining for BioMEMS: review of techniques and examples of applications in microfluidics and drug delivery. Adv Drug Deliv Rev 56:145-172 